\title{
Uma análise acerca das características da síndrome da morte súbita do lactente: revisão bibliográfica
}

\author{
An analysis of the characteristics of sudden infant death syndrome: a literature review
}

Un análisis de las características del síndrome de muerte súbita del lactante: una revisión de la literatura

Tarcila Silveira de Paula Fonseca ${ }^{1 *}$, João Pedro Franco Cerqueira1, Maria Thereza Castilho dos Santos ${ }^{1}$, Gabriel de Lima Machado da Fonseca1, Marianna Ramalho de Sousa1, Pedro Henrique Almeida Warol'1, Emílio Conceição de Siqueira'.

\section{RESUMO}

Objetivo: Revisar e analisar as características da síndrome da morte súbita do lactente. Revisão bibliográfica: A Síndrome da Morte Súbita Infantil (SMSI) é a morte súbita e inexplicada de uma criança com menos de um ano de idade, que permanece inexplicável mesmo após uma autópsia, investigação detalhada da cena da morte e análise da história clínica. A etiologia é baseada por um modelo de "risco triplo" que postula a ocorrência em uma criança biologicamente vulnerável durante um período crítico de desenvolvimento, quando desencadeada por um estressor. Existem fatores de risco não modificáveis como o baixo peso ao nascer e modificáveis como o tabagismo materno, a posição de dormir e a presença de lençóis e colchão macios para criança dormir. Estando a prevenção condicionada a modificação dos fatores de risco. Considerações finais: Nesse contexto, pôr na maioria das vezes ser uma causa de morte evitável, é essencial a atuação na prevenção com a disseminação de informações para os profissionais de saúde para que esses possam conscientizar as famílias sobre a importância da posição prona e superaquecimento, além do controle do tabagismo materno.

Palavras-chave: Morte súbita do lactente, Mortalidade infantil, Criança.

\begin{abstract}
Objective: To review and analyze the characteristics of sudden infant death syndrome. Bibliographic review: Sudden Infant Death Syndrome (SIDS) is the sudden, unexplained infant death syndrome of a oneyear-old child, which remains unexplained even after a detailed death scene investigation and clinical history analysis. The strategy is based on a triple risk model that postulates occurrence at a critical period of biologically vulnerable development, when triggered by a stressor. There are non-modifiable risk factors such as low weight and modifiable risk factors such as maternal sleeping or sleeping, sleeping position with the presence of soft sheets and pillows for a child. The condition of prevention being the modification of risk factors. Final considerations: In this, in most cases, being a preventable cause of death, it is essential to act in prevention with the dissemination of health professional information so that they can make families aware of the importance of the prone position and overheating, in addition to controlling the maternal smoking.
\end{abstract}

Keywords: Sudden infant death, Infant mortality, Child.

\section{RESUMEN}

Objetivo: Revisar y analizar las características del síndrome de muerte súbita del lactante. Revisión bibliográfica: El Síndrome de Muerte Súbita del Lactante (SMSL) es el síndrome de muerte infantil súbita e inexplicable de un niño de un año de edad, que permanece sin explicación incluso después de una investigación detallada de la escena de la muerte y un análisis de la historia clínica. La estrategia se basa en un modelo de triple riesgo que postula la ocurrencia en un período crítico de desarrollo biológicamente vulnerable, cuando se desencadena por un factor estresante. Existen factores de riesgo no modificables

1 Universidade de Vassouras (UV), Vassouras - RJ. *E-mail: tarcila25@hotmail.com 
como el bajo peso y factores de riesgo modificables como el sueño materno o sueño, posición para dormir con presencia de sábanas suaves y almohadas para un niño. Siendo la condición de la prevención la modificación de los factores de riesgo. Consideraciones finales: En esta, en la mayoría de los casos, al ser una causa de muerte prevenible, es fundamental actuar en prevención con la difusión de información a los profesionales de la salud para que puedan concienciar a las familias sobre la importancia del decúbito prono y el sobrecalentamiento, además al control del tabaquismo materno.

Palabras clave: Muerte súbita del lactante, Mortalidad infantil, Niño.

\section{INTRODUÇÃO}

Nos primeiros 12 meses de sua vida, a criança passa mais da metade do tempo dormindo. Este sono não representa somente uma condição de repouso, mas um estado que envolve intensa atividade cerebral e que está diretamente relacionado ao desenvolvimento cognitivo, psicomotor e temperamental da criança (OLIVEIRA AMF, et al., 2020).

Embora essencial à vida humana e, sobretudo, fundamental para o desenvolvimento infantil, alguns hábitos de sono caracterizam-se, no primeiro ano de vida, como fatores de risco para a ocorrência da Síndrome da Morte Súbita do Lactente (SMSL), condição de grande impacto nos indicadores de mortalidade infantil em países desenvolvidos (KONSTAT-KORZENNY E, et al., 2019).

A síndrome da morte súbita infantil (SMSI) é a morte súbita e inexplicada de uma criança com menos de um ano de idade, que permanece inexplicável mesmo após uma autópsia, investigação detalhada da cena da morte e análise da história clínica. A SMSL é a causa mais frequente de morte no período pós-neonatal, com taxa de mortalidade de 0,64-2 por 1.000 nascidos vivos, dos quais $90 \%$ morrem nos primeiros 6 meses de vida (MIRONOVA DIE, et al., 2021).

O principal modelo etiológico da SMSL é o modelo de "risco triplo" que postula que a SMSL ocorre em uma criança biologicamente vulnerável durante um período crítico de desenvolvimento, quando desencadeada por um estressor (KEYWAN C, et al., 2021).

Embora evidências recentes tenham sugerido que apenas 10\% dos casos de SMSL hospedam variantes patogênicas prováveis em genes associados à canalopatia, essa associação representa uma suscetibilidade subjacente a arritmias cardíacas em alguns bebês com SMSI. Ademais, existem disparidades raciais, socioeconômicas e ambientais conhecidas na incidência de SMSL, incluindo raça negra, cama compartilhada, cama macia, superfície para dormir, prematuridade, tabagismo, peso ao nascer, posição de dormir, renda, ocupação, educação e moradia (YAMADA MM, et al., 2021).

A mortalidade infantil é um grave problema de saúde pública mundial e a síndrome da morte súbita do lactente é uma das principais causas de mortalidade de crianças no período pós-natal. Nesse sentido, tornase essencial os estudos de epidemiologia, fatores de risco e fatores de proteção visando a difusão de informações sobre a síndrome e, assim, a sua prevenção. $O$ objetivo do estudo foi revisar e analisar as características da síndrome da morte súbita do lactente.

\section{REVISÃO BIBLIOGRÁFICA}

\section{Definição}

Morte súbita (MS) descreve um evento fatal súbito e não traumático que ocorre na primeira hora após o início ou o agravamento de sintomas em indivíduos aparentemente saudáveis ou, caso não seja testemunhado, dentro de 24 horas depois do indivíduo ter sido visto em boa saúde. Se a MS envolver um lactente com idade inferior a um ano e em um evento em que não foi possível obter uma explicação, mesmo depois de uma exaustiva investigação, a qual inclui autópsia, investigação do local onde ocorreu o óbito e a revisão da história clínica, este evento é descrito como a SMSL (MACHADO PFA, et al., 2018; LIBMAN P, et al., 2021).

A SMSL é caracterizada pela morte súbita de uma criança aparentemente saudável, tipicamente associada a um período de sono, durante o próprio sono ou durante um dos muitos despertares espontâneos que ocorrem a partir do sono (KINNEY HC e HAYNES RL, 2019). 
A síndrome é resultado da combinação de fatores intrínsecos e extrínsecos que se sobrepõem durante um período de desenvolvimento respiratório, autonômico e cardíaco, geralmente ocorrendo entre dois a quatro meses de idade, leva a um evento com risco de vida durante o período de sono. A falha dos mecanismos de proteção durante esses episódios termina finalmente com a morte inesperada (PERRONE S, et al., 2021).

Atualmente, considera-se uma origem multifatorial e que ocorra quando existe um lactente vulnerável, num período de desenvolvimento crítico e instável no que concerne o controle homeostático (o período de maior risco é de 2 a 4 meses, com $90 \%$ dos casos ocorrendo antes dos 6 meses), e que experimente um agente estressor exógeno (MATOSO LML, 2019; PARATZ ED, et al., 2020).

\section{Epidemiologia}

Cerca de $90 \%$ dos casos ocorrem nos primeiros seis meses de idade, sendo o pico apontado entre dois e quatro meses, com posterior declínio, pois é neste período que os bebês aprendem a virar a cabeça, fazendo com que a ocorrência de SMSL torna-se menos frequente (LIBMAN P, et al., 2021; MIRONOVA DIE, et al., 2021). A prevalência de SMSL é maior em meninos do que em meninas, na proporção de 3:2 (PERRONE S, et al., 2021).

A incidência da síndrome é ligeiramente aumentada em bebês prematuros, com baixo peso e cujas mães possuem menos de 20 anos (MARTINS MEP, et al., 2018). A maioria dos eventos ocorre na casa da criança; as mortes fora de casa são mais frequentes na casa de um familiar ou em uma creche, especialmente se a criança dorme em decúbito ventral e em carrinho e/ou cadeirinha (PERRONE S, et al., 2021; PARATZ ED, et al., 2020).

O diagnóstico de SMSL depende da qualidade da infraestrutura social, o que inclui a responsabilidade e capacidade dos profissionais relacionados para averiguar os casos de bebês que falecem subitamente por motivos não conhecidos. Alguns países relataram estimativas bastante precisas da incidência de SMSL, incluindo 3.600 casos anuais nos EUA e 200 casos por ano no Reino Unido. Nos Estados Unidos da América, representa a primeira causa de morte em crianças com idade entre 1 e 12 meses (OLIVEIRA AMF, et al., 2020; MIRONOVA DIE, et al., 2021).

Na Coréia, o Serviço Forense Nacional relatou taxas de 0,2 ou $0,22-0,36$ por 1.000 nascimentos com base em autópsias (AHN YM, et al., 2021). A taxa de SMSL foi estimada em 19,8 por 100.000 nascidos vivos entre 14 países europeus entre 2005 e 2015, variando de 1,4 a 29,2 entre países (JULLIEN S, 2021). $\mathrm{Na}$ Itália, a incidência é de cerca de 1 em cada 1.000 nascidos vivos (PERRONE S, et al., 2021).

No Brasil, a síndrome está numa lista de mortes por causas evitáveis e no ano de 2012 notificou-se 207 óbitos por SMSL, sendo um país no qual as informações acerca do assunto são desencontradas, mas sabese que a incidência é alta (BEZERRA MAL, et al., 2015; MARTINS MEP, et al., 2018).

Nos países ocidentais a mortalidade por SMSL atingiu o pico na década de 1980 e diminuiu durante a década de 1990. Isso ocorreu principalmente devido às campanhas 'Back toSleep', as quais promoveeram a posição de dormir em decúbito dorsal. Nos Estados Unidos, por exemplo, o NationallnfantSleep Position Study demonstrou um aumento na posição supina do sono de 17\% em 1993 para 72\% em 2007(MULLERNORDHORN J, et al., 2020).

Ao lado das recomendações de prevenção da SMSL, a assistência perinatal passou por inúmeras outras melhorias, por isso é difícil atribuir a queda na incidência da síndrome apenas à prática do sono supino (PERRONE S, et al., 2021).

\section{Etiologia}

A etiologia da síndrome é multifatorial, pois cerca de $88 \%$ dos casos de morte súbita infantil envolve dois ou mais fatores (MARTINS MEP, et al., 2018). As mutações em genes associados a síndromes arritmogénicas hereditárias (por exemplo, canalopatias que causam alterações no sistema de condução eléctrico) e a cardiomiopatias (doenças estruturais cardíacas desencadeadas por mutações em genes que codificam vários tipos de proteínas, como, por exemplo, sarcómeros, desmossomas, o citoesqueleto e a membrana nuclear) foram propostas como sendo o substrato para a vulnerabilidade intrínseca dos lactentes vítimas de SMSL (MOREIRA MVF, et al., 2018; PARATZ ED, et al., 2020). 
Até o momento o avanço mais importante foi a descoberta de que a morte acontece durante o período do sono ou nos momentos de transição entre sono e vigília, que se sucedem durante a noite (MATOSO LML, 2019; JOHANNSEN EB, et al., 2021). Distúrbios cardíacos têm sido amplamente investigados como potenciais causas de SMSI, mas ainda há muito que é desconhecido nessa área. Bebês com síndromes de arritmia hereditária podem morrer de parada cardíaca súbita por taquicardia ventricular e/ou fibrilação ventricular. Os procedimentos normais de autópsia não podem detectar essas alterações, uma vez que o coração é estruturalmente normal (MATOSO LML, 2019; MACHADO PFA, 2018).

Estudos genéticos em coortes de SMSI sugerem coletivamente que até $15 \%$ dos casos de SMSI podem ser explicados por doenças cardíacas hereditárias não detectáveis durante investigações convencionais de autópsia forense. As canalopatias iônicas, como a Síndrome de Brugada (SB), a Síndrome do QT Longo (SQTL), a síndrome do QT curto ou a taquicardia ventricular polimórfica catecolaminérgica (TVPC), são descritas como funções interrompidas dos canais, causando distúrbios no fluxo de corrente iônica e arritmias cardíacas letais (ANDERSON TM, et al., 2019).

As arritmias cardíacas, em particulara SQTL, são um mecanismo plausível que contribui para as mortes por SMSL. A SQTL é herdada de forma autossômica dominante caracterizada por intervalos QT prolongados em eletrocardiogramas (ECG) quando causas secundárias foram excluídas. Pacientes com SQTL apresentam risco aumentado de síncope e morte súbita cardíaca (MSC) (KEYWAN C, et al., 2021; PARATZ ED, et al., 2020).

Além disso, doenças metabólicas hereditárias não diagnosticadas, como deficiência de Acetil-CoA desidrogenase de cadeia média (MCAD) ou deficiência do metabolismo da glicose, podem contribuir para a causa da morte em outro 1\% dos bebês com SMSL (NEUBAUER J, et al., 2017; MCGUONE D, et al. 2020; JOHANNSEN EB, et al., 2021).

Quanto à neuropatologia da SMSL, esta se baseia principalmente na avaliação do tronco encefálico, em decorrência do seu papel crítico na regulação do sistema nervoso autônomo. O sistema serotoninérgico do tronco encefálico (5-HT) regula a respiração e a permeabilidade das vias aéreas com regulação de funções homeostáticas como o sono e o despertar. Foi identificada gliose do tronco cerebral o que representa uma resposta geral (muitas vezes crônica) a uma lesão cerebral e, dependendo do contexto, induz vias protetoras ou prejudiciais para modular a função neurológica (BLACKBURN J, et al., 2020; KINNEY HC e HAYNES RL, 2019; MCGUONE D, et al. 2020).

\section{Fatores de risco e de proteção}

Estudos demonstraram que deitar o lactente de bruços triplica o risco de ocorrência da SMSL. Essa descoberta no início da década de 90 levou a campanhas nacionais e internacionais que defendiam uma posição de suspensão supina para bebês. Desde então, estima-se que as taxas da SMSL tenham diminuído em mais de $50 \%$. Bebês não acostumados a dormir em posição prona e que adotam esta posição apresentam um risco elevado (MATOSO LML, 2019; KINNEY HC e HAYNES RL, 2019).

As posições de dormir não supina ou propensas estão associadas a um maior risco de SMSL. Um estudo que analisou 11.717 casos de óbito infantil, no qual 27,4\% classificados como SMSL, dormir de bruços foi relatado em até $42,4 \%$ dos óbitos. Um estudo recente reuniu informações polissonográficas, documentando que lactentes durante o sono em decúbito ventral apresentavam maior frequência cardíaca, diminuição da saturação de oxigênio e mais tempo com saturação de oxigênio abaixo de $90 \%$, em comparação com lactentes que dormiam em decúbito dorsal, oferecendo possíveis explicações por trás da fisiopatologia da posições inadequadas para dormir e sua relação com a síndrome (KONSTAT-KORZENNY E, et al., 2019; GOLDWATER PN e OBERG EO, 2021).

A posição supina sustentada combinada com habilidades motoras restritas levam à plagiocefalia postural. Além disso, a posição prona propicia o desenvolvimento da força da cintura escapular superior. Dessa forma, embora não haja dados que sustentem essa recomendação e estabeleçam sua frequência e duração, os especialistas recomendam uma certa quantidade de posição PRONA enquanto o bebê está acordado e sendo observado (JULLIEN S, 2021; HEALTHFIELD LJ, et al., 2018). 
Alguns fatores de risco modifiváveis e não modificáveis para a ocorrência da síndrome são: travesseiros, colchões ou cobertas muito macios, dormir na mesma cama que os pais ou outras crianças, tabagismo materno durante a gestação e a exposição da criança após o nascimento, uso de álcool e drogas ilícitas durante a gravidez, superaquecimento, cobertura da cabeça, realização de pré-natal de forma tardia ou não acompanhamento de pré natal, baixa idade materna, prematuros e/ou crianças pequenas para idade gestacional e sexo masculino (AHN YM, et al., 2021; MATOSO LML, 2019).

Alguns fatores de risco não modificáveis e inerentes ao lactente são o baixo peso ao nascer e 0 nascimento prematuro. Um estudo demonstrou que bebês prematuros e pequenos para idade gestacional tiveram maior risco para SMSL do que os grandes para a idade gestacional, embora os mecanismos por trás desses fenômenos sejam incertos (KONSTAT-KORZENNY E, et al., 2019).

O tabagismo materno é um dos fatores de risco para a síndrome e isso decorre da associação entre este hábito e anormalidades serotoninérgicas em importantes núcleos do tronco cerebral de crianças com SMSL. Em modelos animais, a nicotina aumenta a liberação de serotonina e altera o disparo de neurônios serotoninérgicos de maneira dose-dependente. O desenvolvimento neuronal serotoninérgico pode ser interrompido pelo tabagismo materno já no primeiro trimestre (ANDERSON TM, et al., 2019; PARATZ ED, et al., 2020).

A superfície macia do sono tem sido apontada como um fator de risco para a SMSL. Um estudo de casocontrole realizado nos EUA entre 260 casos de SMSL e 260 controles vivos pareados mostrou uma associação entre superfície de sono suave e maior risco da síndrome. Colchões macios podem criar uma bolsa ao redor da criança dentro da qual a dispersão de CO2 é limitada, aumentando o risco de reinalação ou asfixia em bebês colocados em decúbito ventral (JULLIEN S, 2021; MULLER-NORDHORN J, et al., 2020; SMITH RW e COLPITTS M, 2020).

O compartilhamento de quarto demonstrou reduzir o risco de SMSL em até $50 \%$. No entanto, o compartilhamento de cama entre pais e bebê permanece altamente controverso. Embora 0 compartilhamento de cama tenha sido associado a um risco aumentado de SMSL, o compartilhamento de cama também foi avaliado para melhorar o apego e a amamentação, considerado um fator de proteção para a síndrome (JULLIEN S, 2021; RUIZ IB, et al., 2020).

A amamentação é um fator de proteção para SMSL. O aleitamento materno exclusivo é recomendado nos primeiros 6 meses de vida, de acordo com as recomendações globais. Um estudo revelou um efeito protetor de qualquer amamentação, qualquer quantidade por qualquer duração(JULLIEN S, 2021; PARATZ ED, et al., 2020).

O compartilhamento do quarto é um fator de proteção para a sindrome. Compartilhamento de quarto não é sinônimo de dormir junto compartilhando a mesma cama. Implica dormir no mesmo quarto, mas em superfícies separadas, embora próximas a elas. Estudos demonstraram que o compartilhamento de quarto pode reduzir o risco de SMSL pela metade. Portanto, este compartilhamento é essencial pelo menos nos primeiros seis meses de vida e recomendado idealmente durante o primeiro ano (KONSTAT-KORZENNY E, et al., 2019; GOLDWATER PN e OBERG EO, 2021).

\section{Uso da chupeta na síndrome: fator protetor ou de risco?}

A chupeta ou chupeta é um dispositivo que é colocado na boca para estimular o comportamento de sucção não nutritiva. A sucção não nutritiva é considerada um reflexo natural para satisfazer a necessidade de contato da criança e pode incluir sucção irrestrita no seio, dedo, chupeta ou outro objeto (PSAILA K, et al., 2017; SMITH RW e COLPITTS M, 2020).

O uso de chupeta em todo o mundo na primeira infância é muito comum, especialmente em países de baixa e média renda, onde as mães acreditam que o uso de chupeta satisfaz a necessidade natural de sucção de seus bebês. As chupetas são usadas para fins como a sucção não nutritiva para o manejo de procedimentos dolorosos e também como prevenção da síndrome da morte súbita do lactente (PSAILA K, et al., 2017; RUIZ IB, et al., 2020). 
Dentre os riscos relacionados ao uso da chupeta, destacam-se dificuldades no aleitamento materno e possibilidade da redução de sua duração; sintomas infecciosos; riscos de acidentes e má oclusão (RUIZ IB, et al., 2020; BLACKBURN J, et al., 2020).

Entre os benefícios relacionados ao uso da chupeta, durante o sono, destacou-se a diminuição da excitação que leva ao despertar do sono da criança; a melhora da capacidade de respiração bucal; a diminuição de sufocamento; a redução da frequência e da duração de refluxos gastresofágicos; o controle cardíaco simpaticovagal e a reposição da autonomia do tônus cardíaco, fatores contribuintes para a diminuição do risco da SMSL. Evidências apontaram para o uso da chupeta como um "fator protetor" contra a síndrome (MOREIRA MVF, et al., 2018; PARATZ ED, et al., 2020). O uso de chupeta durante o sono tem efeito protetor na SMSL (JULLIEN S, 2021; PSAILA K, et al., 2017).

Uma revisão sistemática que incluiu estudos de caso-controle publicados entre 1993 e 2004 demonstrou efeito protetor da chupeta foi demonstrado para o uso habitual de chupeta (AOR 0,71 [IC 95\% 0,59 a 0,85]; 4 estudos) e para uso de chupeta no último sono (AOR 0,39 [IC 95\% 0,31 a 0,50]; 7 estudos). Os autores também estimaram o número necessário para tratar como $2.733(95 \% \mathrm{Cl} 2.416$ a 3.334), o que significa que uma morte por SMSL poderia ser evitada para cada 2.733 bebês que usam chupeta durante o sono (JULLIEN S, 2021).

O uso de chupeta pode resultar em melhor controle autonômico da respiração e estabilidade cardiovascular, pode manter a permeabilidade das vias aéreas em bebês durante o sono no primeiro ano de vida, ou ambos. Uma hipótese alternativa é a possibilidade de que o ambiente de sono do lactente seja alterado pela alça externa da chupeta, o que pode, por sua vez, alterar a configuração da passagem das vias aéreas ao redor da área da boca e do nariz. A passagem alterada das vias aéreas pode ajudar a prevenir a hipóxia acidental resultante de, por exemplo, asfixia por um cobertor ou roupa de cama macia (PSAILA K, et al., 2017; SMITH RW e COLPITTS M, 2020).

Ademais, a entrada sensorial da chupeta é importante para o tônus muscular das vias aéreas superiores no bebê, o que ajuda a manter a permeabilidade das vias aéreas superiores, mantendo a língua para frente por meio de sua protrusão ativa (PSAILA K, et al., 2017; SMITH RW e COLPITTS M, 2020).

\section{CONSIDERAÇÕES FINAIS}

A mortalidade infantil é um desafio global e uma de suas principais causas é a síndrome da morte súbita do lactente que ocorre quando o bebê previamente hígido falece sem que seja conhecida a causa, mesmo após investigação. Nesse contexto, por na maioria das vezes ser uma causa de morte evitável, é essencial a atuação na prevenção com a disseminação de informações para os profissionais de saúde para que esses possam conscientizar as famílias sobre a importância da posição prona e superaquecimento, além do controle do tabagismo materno.

\section{REFERÊNCIAS}

1. AHN YM, et al. Cultural variation in factors associated with sudden infant death during sleep. BMC Pediatr, 2021; 21:443.

2. ANDERSON TM, et al. Maternal Smoking Before and During Pregnancy and the Risk of Sudden Unexpected Infant Death. Pediatrics, 2019; 143(4): e20183325.

3. BEZERRA MAL, et al. Fatores associados ao conhecimento das mães sobre a Síndrome da Morte Súbita do Lactente. Escola Anna Nery, 2015; 19(2): 303- 309.

4. BLACKBURN J, et al. Revisiting the Neuropathology of Sudden Infant Death Syndrome (SIDS). Front Neurol, 2020; 11: 594550.

5. GOLDWATER PN, OBERG EO. Infection, Celestial Influences, and Sudden Infant Death Syndrome: A New Paradigm, 2021; 13(8): e17449.

6. HEALTHFIELD LJ, et al. A Systematic Review of Molecular Autopsy Studies in Suddenl nfant Death Cases. J PediatrGenet, 2018; 7(4):143-149.

7. JOHANNSEN EB, et al. The Genetics of Sudden Infant Death Syndrome-Towards a Gene ReferenceResource. Genes (Basel), 2021; 12(2):216. 
8. JULLIEN S. Sudden infant death syndrome prevention. Jullien BMC Pediatrics, 2021; 21(1):320.

9. KEYWAN C, et al. Genetic Factors Underlying Sudden Infant Death Syndrome. Appl Clin Genet, 2021; 15(14): 61-76.

10. KINNEY HC, HAYNES RL. The Serotonin Brainstem Hypothesis for the Sudden Infant Death Syndrome. J Neuropathol ExpNeurol, 2019; 78(9): 765-779.

11. KONSTAT-KORZENNY $\mathrm{E}$, et al. Morte súbita infantil inesperada: Revisão e análise da adesão às recomendações. Cureus, 2019; 11(11): e6076.

12. LIBMAN $P$, et al. Prevenção de morte súbita em lactentes: uma revisão bibliográfica. REAC, 2021; 35 : e8660.

13. MACHADO PFA. Síndrome da Morte Súbita do Lactente. Monografia (Mestrado Integrado em Medicina). Faculdade de Medicina da Universidade do Porto. Porto, 2018; 45p.

14. MCGUONE D, et al. Sudden Unexplained Death in Childhood: A Neuropathology Review. Front Neurol, 2020; 16(11): 582051.

15. MARTINS MEP, et al. Síndrome da Morte Súbita Infantil (SMSI): aspectos acerca das principais causas e as formas de prevenção. Id onLine Rev. Mult. Psic, 2018; 12(41):192-205.

16. MATOSO LML. Morte súbita do lactente: uma revisão integrativa. Revista Saúde e Desenvolvimento, 2019; 13(16): 74-90.

17. MIRONOVA DIE, et al. Sudden infant death syndrome: Melatonin, serotonin, and CD34 factor as possible diagnostic markers and prophylactic targets. PLoS ONE, 2021; 16(9): e0256197.

18. MOREIRA MVF, et al. Uso de chupetas na prevenção da síndrome da morte súbita do lactente: revisão narrativa da literatura. Rev. APS, 2018; 21(4): 820 - 821.

19. MULLER-NORDHORN J, et al. International time trends in sudden unexpected infant death, 1969-2012. BMC Pediatr, 2020; 20(1): 377.

20. NEUBAUER $\mathrm{J}$, et al. Post-mortem whole-exomeanalysis in a large sudden infant death syndrome cohortwith a focuson cardiovascular and metabolic genetic diseases. Eur J Hum Genet, 2017; 25(4):404-409.

21. OLIVEIRA AMF, et al. Risk and protective factors for sudden infant death syndrome. Revista Brasileira de Enfermagem, 2020; 73(2): e20190458.

22. PARATZ ED, et al. Cardiac arrestand sudden cardiac death registries: a systematic review of global coverage. Open Heart, 2020; 7(1): e001195.

23. PERRONE S, et al. Sudden Infant Death Syndrome: Beyond Risk Factors. Life (Basel), 2021; $11(3): 184$.

24. PSAILA K, et al. Infant pacifiers for reduction in risk of sudden infant death syndrome. Cochrane Database Sys tRev, 2017; 4(4): 011147.

25. RUIZ IB, et al. Síndrome de muerte súbita del lactante: ¿siguen las familias las recomendaciones? An Pediatr (Engl Ed). 2020; 92(4):222-228.

26. SMITH RW, COLPITTS M. Pacifiers and the reduced risk of sudden infant death syndrome. Paediatr Child Health, 2020; 25(4): 205-206.

27. YAMADA MM, et al. Risk Factors for Sudden Infant Death in North Carolina. Front Pediatr, 2021; 9: 770803. 